\title{
Multifunctional Complex of Thermomechanical Calibration by Screw Compression
}

\author{
Nikita Balobanov ${ }^{1, *}$, Vyacheslav Dementyev ${ }^{1}$, Sergey Makarov ${ }^{1}$, Evgeny Suntsov and \\ Alexander Korshunov ${ }^{1}$ \\ ${ }^{1}$ Udmurt Federal Research Center of the Ural Branch of the Russian Academy of Sciences,34, \\ T.Baramzinoy St., Izhevsk,426067, Russia
}

\begin{abstract}
In the present paper, a soft-hardware system is considered intended for controlling the complex for high-temperature thermomechanical calibration by screw compression. The functional capabilities and block diagram of the complex are presented. The operation algorithm is briefly described and the criteria for selecting parameters of processing are given. The machining program structure, data on the operator panel, manual mode and measured parameters of the calibration process are shown.
\end{abstract}

\section{Introduction}

High-temperature thermomechanical processing by screw compression is a method for obtaining axisymmetric metal products from structural steels with improved mechanical and performance characteristics [1,2]. For the realization of the search for optimal parameters and the integrated study of the influence of each selected parameter on the final quality level of hardened metal products, a multifunctional research complex for thermomechanical calibration (Fig.1) has been designed and constructed $[3,4]$.

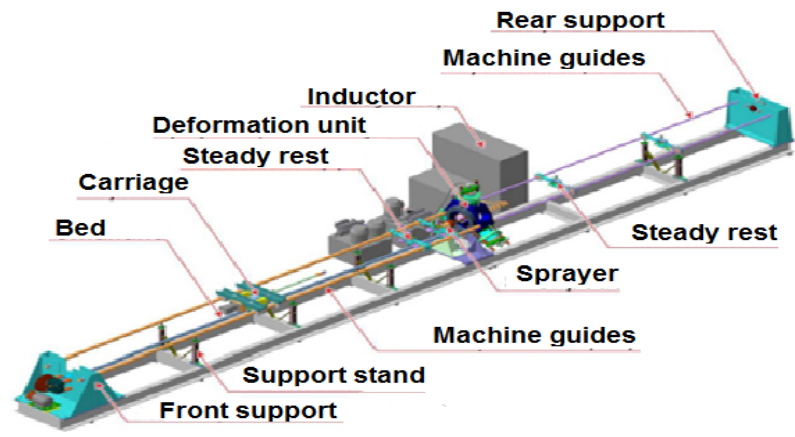

Fig. 1. Block diagram of the complex.

The complex allows the following:

\footnotetext{
* Corresponding author: bna@udman.ru
} 
1. To control the variations of force and kinematic parameters in the coordinates of the carriage movement during the entire cycle of processing. Such parameters are the rotational torque of the gripper rotary drive with an angular velocity of rotation; the axial force of the drive of the gripper linear advance with a speed; the retained force of a mandrel for a hollow workpiece; the radial force acting on rollers; the gripper rotational velocity; the rotational speed of the rollers; axial velocity.

2. To provide the specified force characteristics of the process, the control of the velocities of rotation and linear advancement, the control of post-deformation hold.

3. To create the required modes of cooling: it is done by controlling the cooling medium temperature and the medium consumption in a sprayer

4. To save all the data incoming from a flash memory card with the registration of time or movement for further analysis.

\section{Algorithm of operation and criteria for selecting processing parameters}

In the structure of the control program for the complex the regulated parameters are in the form of sets created for each particular production or research task. Figure 2 shows the list of the parameters of processing entered by an operator on the display of the complex.

\begin{tabular}{|c|c|c|c|c|c|c|c|}
\hline \multicolumn{8}{|c|}{ Current parameters of the complex } \\
\hline \multicolumn{4}{|c|}{ "Name of the parameter set" } & \multirow{2}{*}{\begin{tabular}{|l|} 
a1 \\
a7 \\
\end{tabular}} & \multirow{2}{*}{$\begin{array}{l}\text { Initial carrage position, } \\
\text { Compression start point, }\end{array}$} & \multirow{2}{*}{$\mathrm{mm}$} & \multirow{2}{*}{$\begin{array}{l}0,00 \\
0,00\end{array}$} \\
\hline s1 & Carriage speed, & $\mathrm{mm} / \mathrm{sec}$ & 0,0000 & & & & \\
\hline 81 & Workpiece rotation speed, & rpm & 0 & as & Compression end point, & $\mathrm{mm}$ & 0,00 \\
\hline $\mathbf{a 3}$ & Start point of rotation, & $\mathrm{mm}$ & 0,00 & a9 & Irrigation start point, & mm & 0,00 \\
\hline a4 & End paint of rotation, & $\mathrm{mm}$ & 0,00 & a10 & Irrigation end point, & $\mathrm{mm}$ & 0,00 \\
\hline a5 & Initial heating point, & mm & 0,00 & a11 & Steady-rest entry point, & mm & 0,00 \\
\hline \multirow[t]{2}{*}{ a6 } & Heating end point, & $\mathbf{m m}$ & 0,00 & a12 & Steady-rest output point, & $\mathrm{mm}$ & 0,00 \\
\hline & \begin{tabular}{|l|l|} 
Sprayer & water \\
2 water + air
\end{tabular} & & 1 & a2 & Carriage end point, & $\mathrm{mm}$ & 0,00 \\
\hline \multicolumn{7}{|c|}{ Current carriage position $\quad 0,00$} & \\
\hline
\end{tabular}

Fig. 2. Photo of the display of the complex

It is possible to activate the manual mode by pressing the corresponding buttons on the panel (Fig. 3). The initial position of the carriage is selected by moving it manually to the required coordinate at visual control. 


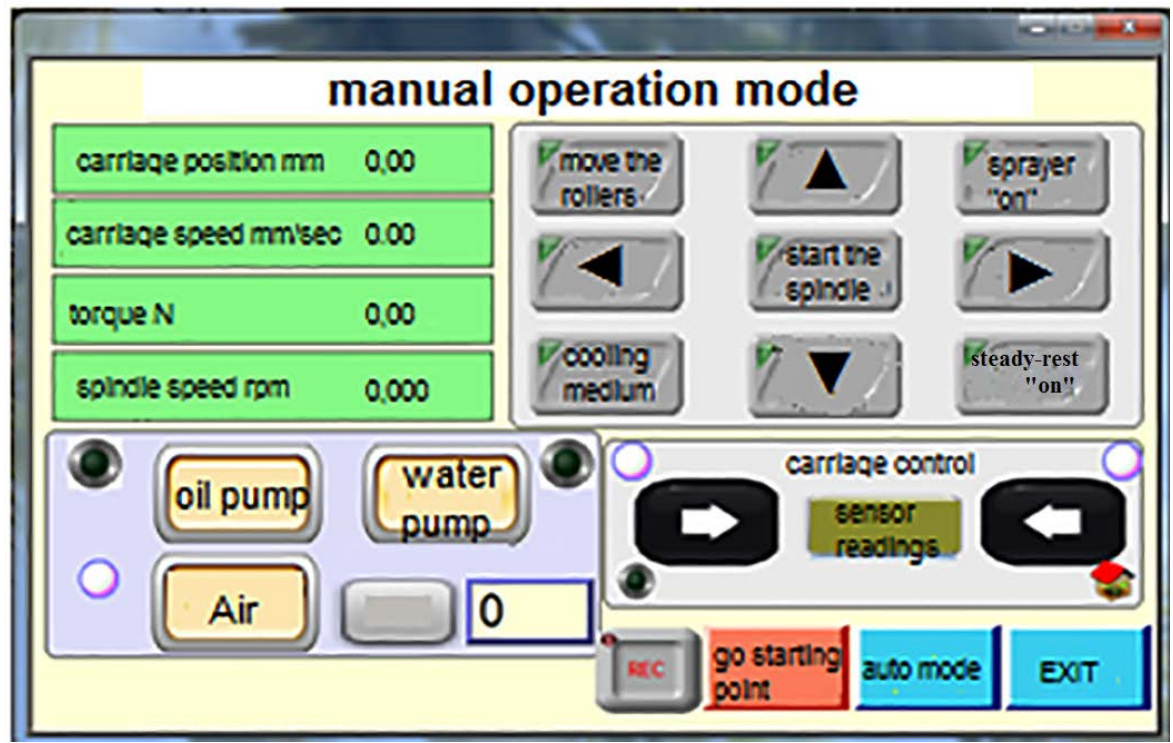

Fig. 3. The "manual operation mode" tab

In the general case, the algorithm of making a set of the parameters of processing includes the following operations:

1. The capacity of the heating unit is determined.

2. Based on the input data, the calculation is made of the rotation velocity of a component, the angle of the turn of rollers and the percent increase of the angle of advancement relative to the angle of the roller turn.

3. Using the values of the rotation velocities and speeds of advancement and taking into account real geometry parameters of the processing rollers (the diameter, the width of the conical part and the width of the cylindrical part) and the workpiece, the amount of unit strain on the both parts of the rollers is calculated.

4. The coordinate of the beginning of the operation cycle is entered. Taking into account the lay-out diagram, the above coordinate is selected so that the nonmachining part of the workpiece would be minimized and the gripper would not be heated by the inductor.

5. The operation parameters of the deformation unit are specified.

6. The sprayer parameters are entered.

7. The coordinate of the advancement of the supporting steady-rests is specified.

8. The coordinate of switching the inductor off is specified.

9. Taking into consideration the elongation of the workpiece, the coordinate of the stop of the gripper axial advancement is calculated.

10. The parameters are input in the form of sets on the control panel. The installation is ready for the initiation of the processing cycle.

In the process of adjusting each set, the controller saves the readings of the sensors of the complex onto removable media for providing synchronization with the current coordinate of the carriage in accordance with the periodicity specified by the service engineer of the complex; the time reference to the current moment is fixed. 


\section{Results}

In Fig. 4, one can see the graphical representation of the values of the force parameters: the axial force $F$ on a workpiece, the forces of the roller pressing $F_{1}, F_{2}$ and $F_{3}$ and the axial force on the carriage $F_{c}$ depending on the position of the workpiece in the complex. A workpiece made from structural steel was processed $[5,6]$. On the workpiece section with the length from $0 \mathrm{~mm}$ to $200 \mathrm{~mm}$ the variations of the axial force are due to the transient processes associated with the beginning of the workpiece processing. The length of this section is normally equal to ten diameters of the workpiece. On the section from $200 \mathrm{~mm}$ to $280 \mathrm{~mm}$ the processes take place associated with the beginning of the deforming rollers operation [7]. On the mentioned section, the axial force of the carriage movement sharply decreases as the intensity of plastic deformation decreases. The growth of the axial force on the section from $600 \mathrm{~mm}$ to $680 \mathrm{~mm}$ is associated with the workpiece cooling through the end surface and with the heating of the deforming rollers due to the contact with the workpiece. On the section of $680 \mathrm{~mm}-1040 \mathrm{~mm}$ the axial force decrease takes place due to the end of the deformation. The workpiece is moved to the zone of cooling by the sprayer (the processes in the zone are described in [8-10]) and the workpiece processing comes to an end.

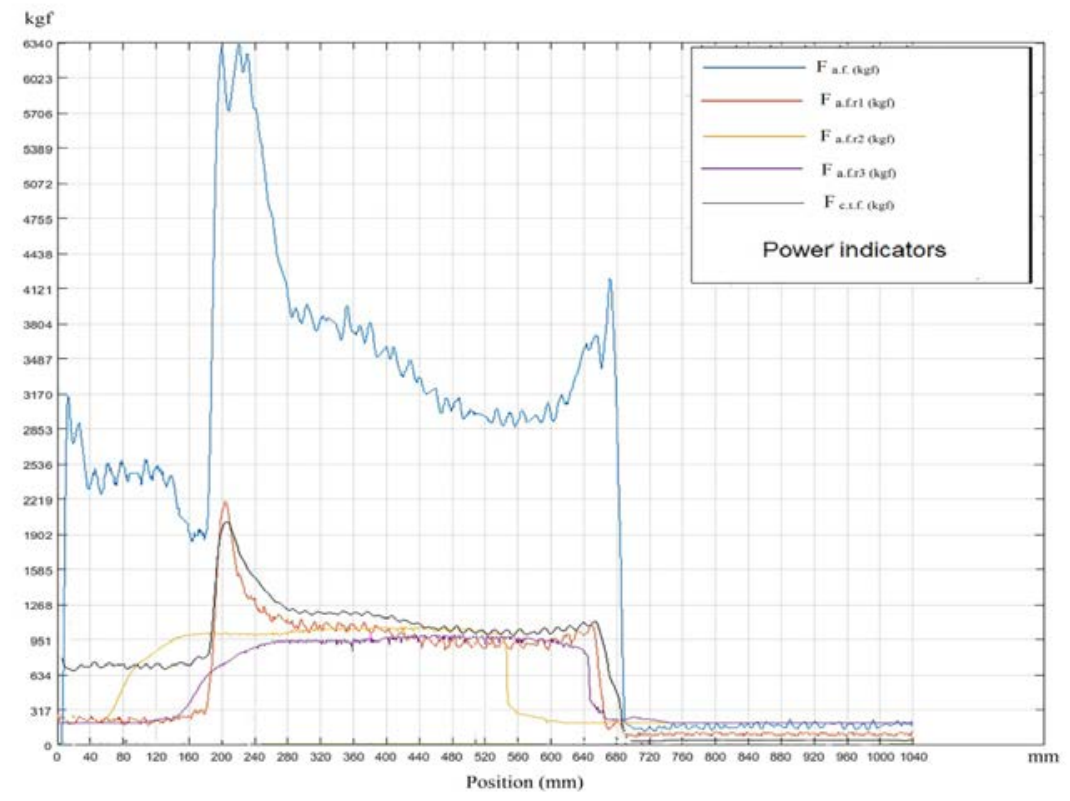

Fig. 4. Power processing parameters of the workpiece

\section{Conclusion}

A multifunctional complex for thermomechanical processing is developed allowing the control of the main force and kinematic parameters of the calibration process by screw compression. The technical regimes form the required structure and mechanical and operating characteristics of components. The complex allows studying the influence of the main process variables on the stress-strain state of workpieces and selecting technical regimes of processing which guarantee obtaining improved physical and mechanical characteristics of material and high quality of metal products. 


\section{References}

1. M. L. Bernstein, V. A. Zaymovsky, L. M.Kaputkina, Thermomechanical processing of Steel, Moscow, Metallurgiya, (1983.)

2. A.V.Trukhachev, A. N. Ilina, A. G. Shchuklin, Investigation of structural changes during hot torsion deformation, Izhevsk, (1974)

3. E. S.Markova, N. G. Pokrovskaya, A. B. Shalkevich, V. I. Gromov, Martensit-aging steels -new promising materials for gas turbine engine shafts, Aviation materials and technologies, (2012)

4. O. I. Shavrin, V. B. Dementyev, V. A. Ivanov Methodology of the study of deformedstate of cylindrical samples by the rod method, Zavodskaya laboriya, № 12 (1983)

5. O. I. Shavrin, V. B. Dementiev, N. A. Kokorin, Technology for obtaining a volumetric coordinate grid, Ferrous metallurgy,(1985)

6. E. A. Ulyanin Corrosion-resistant steels and alloys: Reference book, Moscow, Metallurgy, (1991)

7. N. A.Balobanov, Yu. V. Ganziy The method of repairing the rods of oil pumps with the use of VTMO VO technology, materials of the IX Interregional Scientific and Practical Conference, (2019)

8. S. S. Makarov, M. Yu. Ales, Balobanov N. A. Numerical simulation of conjugate heat transfer during cooling of a high-temperature metal body by a gas-liquid medium flow, Engineering physics, No. 9, (2019).

9. V. B. Dementiev, S. S. Makarov, K. E. Chekmyshev, N. A. Balobanov, The method of hardening metattic products during thermomechanical processing,Patent for the invention RU 2702524 C1, (2019)

10. V. B. Dementiev, A.M. Lipanov, S. N. Khramov A method for creating a cooling medium with controlled thermophysical properties, Patent No. 2354712 Ros. Federation: IPC7 A / IPM of the Ural Branch of the Russian Academy of SciencesNo. 2007125180/02; application No. 03.06.2007; Publ. 10.05.2009 Byul. No. 13. 